\title{
Unified Mobility Model for Disordered Organic Semiconductors
}

\author{
Fabrizio Torricelli, and Luigi Colalongo, Member, IEEE
}

\begin{abstract}
The influence of temperature, carrier density, and electric field on hopping transport in disordered organic semiconductors is discussed, and an accurate mobility model that accounts for all those effects in a single analytical expression is derived. The model is based on the concept of percolation in a variable range hopping system, and the calculations are worked out by exploiting the effective temperature approach. At room temperature, the dependence on carrier density plays a major role, whereas at low temperatures or high fields, the influence of electric field becomes relevant. Neglecting only one of them leads to an evident underestimation of hopping mobility. The model accurately reproduces experiments and numerical simulations and provides, by means of a single mathematical expression, a clear picture of several physical effects as the Poole-Frenkel dependence of the mobility on the electric field or the Arrhenius behavior on temperature.
\end{abstract}

Index Terms-Density of states (DOS), percolation theory, variable range hopping (VRH) transport.

A FTER the discovery of electroluminescence in the disordered conjugated polymer poly-(p-phenylene vinylene) (PPV) and its derivatives, a lot of attention was paid to the study of the optical and electronic properties of conjugated polymers [1]. One of the most important parameters that determine the device performances is the carrier mobility $\mu$. In particular, the dependence of $\mu$ on temperature $T$, electric field $F$, and carrier density has been extensively addressed in the literature [2]-[4], and several models that separately account for each of them were proposed. On the other hand, a clear physical picture of the mobility that globally accounts for the relative effects of temperature, carrier density, and electric field is still absent in the literature.

The aim of this letter is to derive a mobility model that accounts for the most relevant physical quantities, temperature, carrier concentration, and electric field in a single analytical expression. The model is based on the percolation in a variable range hopping (VRH) system with an exponential distribution of localized energy states [density of states (DOS)] [5], while the mathematical calculations are worked out by exploiting the well-accomplished effective temperature approach [6]-[9]. It accurately reproduces experiments and numerical simulations and provides, by means of a single mathematical expression, a clear picture of several effects as, for instance, the

Manuscript received May 28, 2009; revised July 6, 2009. First published September 1, 2009; current version published September 29, 2009. The review of this letter was arranged by Editor M. Ostling.

The authors are with the Department of Electronics for Automation, University of Brescia, 25123 Brescia, Italy (e-mail: fabrizio.torricelli@ing.unibs.it; luigi.colalongo@ing.unibs.it).

Digital Object Identifier 10.1109/LED.2009.2027998
Poole-Frenkel-like dependence of the mobility on the electric field or the Arrhenius dependence on temperature. We expect that this mobility theory enables predictive models for disordered organic devices based on parameters that may be determined $a b$ initio from measurements and quantum mechanical simulation. Owing to its simple analytical formulation, the relative importance of temperature, carrier density, and electric field could singularly investigated and disentangled on the experimental results.

The charge transport in disordered organic semiconductors is governed by hopping between localized states. The conductance between two sites is proportional to the occupation probability of the site that releases the carrier, called donor, the occupation probability of the site that receives the carrier, called acceptor, and the hopping rate between the two sites

$$
G_{i j}=G_{0} f\left(E_{i}, E_{F}\right)\left[1-f\left(E_{j}, E_{F}\right)\right] \nu_{i j} .
$$

The hopping rate from a donor at energy $E_{i}$ to an acceptor at energy $E_{j}$ at a distance $r_{i j}$ may be described by the Miller-Abrahams rate model [10]

$$
\nu_{i j}=\nu_{0} \exp \left[-2 \alpha r_{i j}\right] \exp \left[-\frac{E_{j}-E_{i}}{K_{B} T} \theta\left(E_{j}-E_{i}\right)\right]
$$

where $\nu_{0}$ is the attempt-to-escape frequency, $\alpha^{-1}$ is the localization radius of a charge carrier, $K_{B}$ is the Boltzmann constant, $T$ is the temperature, and $\theta$ is the Heaviside function. Following the study of Vissenberg and Matters [5], at low carrier densities, the transport properties are determined by the tail of an exponential density of the localized states

$$
g(E)=\frac{N_{t}}{k_{B} T_{0}} \exp \left(\frac{E}{k_{B} T_{0}}\right) \quad(-\infty<E<0)
$$

where $N_{t}$ is the total number of states per unit volume, $T_{0}$ is a parameter related to the system disorder, and $g(E)=0$ when $E>0$. Since, in equilibrium, the occupation probability of the localized states can be expressed by the Fermi-Dirac distribution function, the fraction of localized states occupied by carriers reads

$$
\begin{aligned}
\delta N_{t} & =\int_{-\infty}^{+\infty} g(E) f\left(E, E_{F}\right) d E \\
& \simeq N_{t}\left(\frac{\pi T / T_{0}}{\sin \left(\pi T / T_{0}\right)}\right) \exp \left[\frac{E_{F}}{K_{B} T_{0}}\right]
\end{aligned}
$$

where $\delta \in[0,1]$ and $E_{F}$ is the Fermi energy level. The transport is governed by the hopping of carriers between localized states that, in turn, is strongly dependent on the hopping distances 
as well as on the DOS. The system can be described as a resistor network [11], and one may assign a conductance $G_{i j}=G_{0} \exp \left[-s_{i j}\right]$ between site $i$ and site $j$ where

$$
s_{i j}=2 \alpha r_{i j}+\frac{\left|E_{i}-E_{F}\right|+\left|E_{j}-E_{F}\right|+\left|E_{i}-E_{j}\right|}{2 K_{B} T} .
$$

It is worth noting that in the aforementioned expression of $s_{i j}$, adopted by Vissenberg and Matters [5], it is implicitly assumed that $\left|E-E_{F}\right|>2 K_{B} T$; in other words, they assume a rather low carrier concentrations. Therefore, the Fermi-Dirac distribution is accurately approximated by the Boltzmann one, and the expression of $s_{i j}$ may be simplified as

$$
s_{i j}=2 \alpha r_{i j}+\left\{\begin{array}{ll}
\frac{E_{j}-E_{F}}{K_{B} T}, & E_{j}>E_{i} \\
\frac{E_{i}-E_{F}}{K_{B} T}, & E_{i} \geq E_{j}
\end{array} .\right.
$$

It leads to a very simple integration domain, and the percolation criterion is straightforwardly calculated without introducing any simplification with respect to the study in [5]. The modified integration domain is the key point to work out a simple and accurate analytical expression of the mobility in the presence of electric field. According to the percolation theory [11], the conductivity of the system could be expressed as $\sigma=\sigma_{0} \exp \left(-s_{c}\right)$, where $\sigma_{0}$ is a prefactor (to be calculated) and $s_{c}$ is the exponent of the critical percolation conductance. The onset of percolation is determined by calculating the critical average number of bonds per site

$$
B\left(G=G_{c}\right)=B_{c}=\frac{N_{B}\left(s_{c}, E_{F}\right)}{N_{s}\left(s_{c}, E_{F}\right)}
$$

where $B_{c}=2.8$ for a 3-D amorphous system [12] and $N_{b}$ and $N_{s}$ are the density of bonds and the density of sites in a percolation system, respectively. The total density of bonds can be calculated by integrating in energy, over the distance $r_{i j}$, the product of all the available sites (at energy $E_{i}$ ) and of all the available target states (at energy $E_{j}$ ) that satisfy the percolation criterion

$$
N_{B}=4 \pi \int_{\mathbb{R}^{3}} r_{i j}^{2} g\left(E_{i}\right) g\left(E_{j}\right) \theta\left(s_{c}-s_{i j}\right) d E_{j} d E_{i} d r_{i j} .
$$

The density of sites $N_{s}$ that satisfy the percolation criterion reads

$$
N_{s}=\int_{\mathbb{R}} g(E) \theta\left(s_{c} K_{B} T-\left|E-E_{F}\right|\right) d E .
$$

Furthermore, in the presence of an electric field $\mathbf{F}$, the potential energy is modified, and the Miller-Abrahams hopping rate turns out to be

$$
\begin{aligned}
\nu_{i j} & =\nu_{0} \exp \left[-2 \alpha r_{i j}\right] \\
& \times \exp \left[-\frac{E_{j}-E_{i}-\mathbf{F} \cdot \mathbf{r}_{i j}}{K_{B} T} \theta\left(E_{j}-E_{i}-\mathbf{F} \cdot \mathbf{r}_{i j}\right)\right] .
\end{aligned}
$$

It was demonstrated [9] that the effect of the electric field on the transport coefficients of disordered organic semiconductors may be accurately described by replacing the lattice temperature in the expression of the low-field mobility with an effective temperature $T_{F}(T, F)$ that, in turn, is a function of the field. In the presence of the field, the number of sites available for transport is essentially enhanced in the direction of the field, and the distance between sites, available for hopping, is reduced. Therefore, the nonequilibrium occupation probability should be modified to account for the local variation of $E_{F}$ arising from the external electric field, and the Fermi-Dirac distribution should be generalized to nonequilib$\operatorname{rium} f\left(E, E_{F}\right)=\left[1+\exp \left(E-E_{F} / K_{B} T_{F}\right)\right]^{-1}$, where $T_{F}=$ $\left[T^{\beta}+\left(\gamma e F / \alpha K_{B}\right)^{\beta}\right]^{1 / \beta}$, $e$ is the electron charge, and $\beta$ and $\gamma$ are fitting parameters [9]. Owing to the simplifications introduced with (6), in the presence of electric field, $s_{i j}$ may be worked out

$$
s_{i j}=2 \alpha r_{i j}+ \begin{cases}\frac{\frac{T_{F}}{T} E_{j}^{\prime}-E_{F}+\frac{T-T_{F}}{T} E_{i}}{K_{B} T_{F}}, & E_{j}^{\prime}>E_{i} \\ \frac{E_{i}-E_{F}}{K_{B} T_{F}}, & E_{j}^{\prime} \leq E_{i}\end{cases}
$$

where the electric field is assumed in the $y$-direction (same considerations hold for any other direction) and $E_{j}^{\prime}=E_{j}-$ $e|\mathbf{F}| \sin (\theta) \sin (\varphi) r_{i j}$. Therefore, after straightforward calculations, the percolation criterion in the presence of an external electric field turns out to be

$$
B_{c} \approx \frac{\pi N_{t} T_{0}^{3}}{(2 \alpha)^{3} T_{F}^{2}\left(2 T_{F}-T\right)} \exp \left(\frac{E_{F}+K_{B} T_{F} s_{c}}{K_{B} T_{0}}\right) .
$$

Combining the expression of the system conductivity with (12), the expression of the carrier mobility as a function of temperature, carrier density, and electric field eventually reads

$$
\mu=\frac{\sigma_{0}}{e}\left[\frac{T_{0}^{4} \sin \left(\pi \frac{T}{T_{0}}\right)}{B_{c}(2 \alpha)^{3} T_{F}^{2}\left(2 T_{F}-T\right) T}\right]^{\frac{T_{0}}{T_{F}}}\left(\delta N_{t}\right)^{\frac{T_{0}}{T_{F}}-1} .
$$

It is worth noting that if $\mathbf{F} \rightarrow 0, T_{F} \rightarrow T$, and the aforementioned equation turns out to be exactly the same of [5], hence the two mobility models have exactly the same behavior with respect to the temperature and carrier density. In order to investigate the accuracy of our model when $F>0$, the mobility is compared with the numerical solution of the Pauli master equation [13] that has been proved to accurately reproduce the current-voltage characteristics of both lightemitting diodes and field-effect transistors. In particular, in [13], the numerical mobility that we use to check our model was successfully tested on hole-only diode devices of poly[ $40^{\prime}-(3$, 7-dimethyloctyloxy)-1, 1'-biphenylene-2, 5-vinylene] (NRSPPV), with layer thickness $L$ of $560 \mathrm{~nm}$, and poly(2-methoxy5-(3', 7'-dimethyloctyloxy)-p-phenylene vinylene) $\left(\mathrm{OC}_{1} \mathrm{C}_{10}-\right.$ PPV), with $L$ of $275 \mathrm{~nm}$ with the following fitting parameters: $c_{1}=1.8 \times 10^{-9}, c_{2}=0.42$, and $\sigma=0.14 \mathrm{eV}$. In Fig. 1, the mobility as a function of the electric field is reported at different temperatures for a carrier density $\delta N_{t}=3 \cdot 10^{16} \mathrm{~cm}^{-3}$ (LED devices). The parameters of our model $\left(N_{t}=1.7 \cdot 10^{20} \mathrm{~cm}^{-3}\right.$, $T_{0}=400 \mathrm{~K}, \sigma_{0}=10^{4} \mathrm{~S} / \mathrm{cm}, \alpha^{-1}=1.8 \AA, \beta=1.8$, and $\gamma=$ $0.62)$ are in a very good agreement with others reported in literature: $N_{t}$ and $\alpha$ are the same as those in [13], $T_{0}$ is derived by approximating the Gaussian DOS as reported in [14], and $\gamma$ and $\beta$ are fitting parameters in perfect agreement with those in [9]. The two models are in perfect agreement, and when 


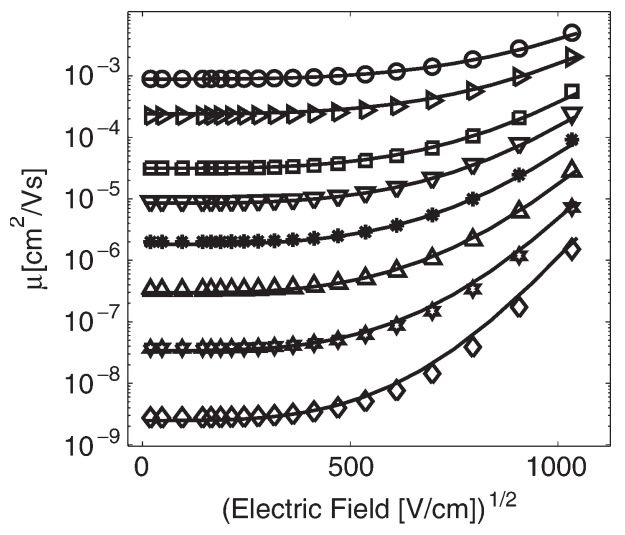

Fig. 1. (Symbols) Numerical and (lines) analytical mobilities as a function of the electric field at different temperatures for NRS-PPV. $\diamond T=180 \mathrm{~K}$, $\star T=200 \mathrm{~K}, \triangle T=220 \mathrm{~K}, * T=240 \mathrm{~K}, \nabla T=260 \mathrm{~K}, \square T=280 \mathrm{~K}$, $\triangleright T=320 \mathrm{~K}$, and $\circ T=360 \mathrm{~K}$.

$F>3 \times 10^{5} \mathrm{~V} / \mathrm{cm}$, the well-known Pool-Frenkel behavior $\mu \propto \exp [\gamma \sqrt{F}]$ is correctly reproduced. Hence, as reported in [13], there is no need to assume spatial energy correlation, as suggested by several authors, to explain the electric field dependence, and the sites may be assumed uncorrelated. It is worth adding that a satisfactory description of the mobility as a function of the field is achieved by considering multiphonon hop on an exponential DOS [5], where the energy is provided to the system by the electric field as well. In the presence of the electric field, the number of sites available for charge transport is essentially enhanced in the direction of the field. The distance between sites available for hopping transport shortens; hence, the carriers can move faster. It is particularly clear at low temperature, as shown in the figures; the dependence on the electric field, in fact, is much more pronounced as the thermal energy is progressively less prevailing with respect to the potential energy arising from the electric field. As reported in [14], the mobility is strictly correlated to the carrier density that, in turn, depends on the shape of the DOS. It follows that our model correctly approximates the numerical solution of [13] that is based on a Gaussian DOS, only locally. Therefore, the characteristic parameters of the exponential distribution, i.e., $N_{t}$ and $T_{0}$, should be slightly modified when the mobility is calculated at large carrier concentrations (FET devices) or at low carriers concentrations (LED devices) [14]. In Fig. 2, the mobility as a function of temperature is compared with that in [13] as well. The two models are in perfect agreement; furthermore, the mobility exhibits a clear Arrhenius-like temperature behavior $(\log (\mu) \propto$ $1 / T)$, and it is consistent with the data published in [15].

To conclude, in this letter, we have presented a unified description of the carrier mobility in disordered organic materials. The model generalizes the well-known approach of Vissenberg and Matters [5], including the effect of the electric field. Following a new straightforward mathematical approach, the effect of the electric field could be easily accounted for in a simple analytical model. The model is based on the concept of percolation in a VRH system, and the effect of the electric field is accounted for by exploiting the effective temperature approach. It accurately reproduces experiments and numerical simulations and provides, by means of a single mathematical

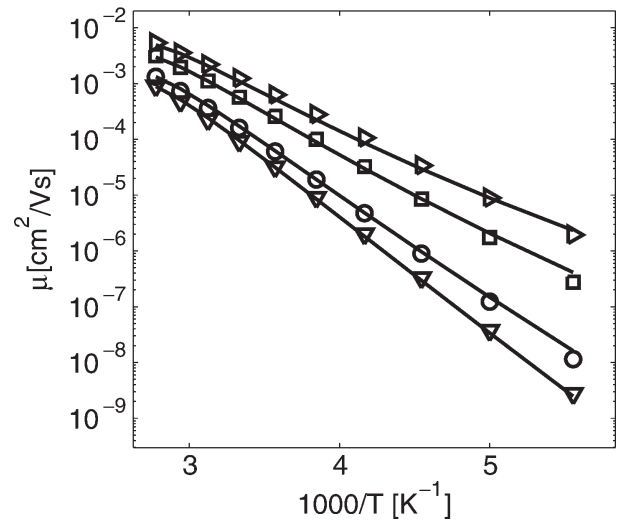

Fig. 2. (Symbols) Numerical and (lines) analytical mobilities as a function of the temperature at different electric fields for NRS-PPV. $\nabla F=0 \mathrm{~V} / \mathrm{cm}$, $\circ F=0.45 \mathrm{MV} / \mathrm{cm}, \square F=0.87 \mathrm{MV} / \mathrm{cm}$, and $\triangleright F=1.1 \mathrm{MV} / \mathrm{cm}$.

expression, a clear picture of several physical effects as the Poole-Frenkel-like dependence of the mobility on the electric field or the Arrhenius behavior on temperature.

\section{REFERENCES}

[1] P. W. M. Blom and M. C. J. M. Vissenberg, "Charge transport in poly(pphenylene vinylene) light-emitting diodes," Mater. Sci. Eng., R Rep., vol. 27, no. 3/4, pp. 53-94, May 2000.

[2] Z. G. Yu, D. L. Smith, A. Saxena, R. L. Martin, and A. R. Bishop, "Molecular geometry fluctuation and field-dependent mobility in conjugated polymers," Phys. Rev. B, Condens. Matter, vol. 63, no. 8, pp. $85202-$ 85210 , Feb. 2001.

[3] J. Cottaar and P. A. Bobbert, "Calculating charge-carrier mobilities in disordered semiconducting polymers: Mean field and beyond," Phys. Rev. B, Condens. Matter, vol. 74, no. 11, pp. 115 204-115 209, Apr. 2006.

[4] B. N. Limketkai, P. Jadhav, and M. A. Baldo, "Electric-field-dependent percolation model of charge-carrier mobility in amorphous organic semiconductors," Phys. Rev. B, Condens. Matter, vol. 75, no. 11, pp. $113203-$ 113 206, Dec. 2007.

[5] M. C. J. M. Vissenberg and M. Matters, "Theory of the field-effect mobility in amorphous organic transistors," Phys. Rev. B, Condens. Matter, vol. 57, no. 20, pp. 12964-12967, Jan. 1998.

[6] S. Marianer and B. I. Shklovskii, "Effective temperature of hopping in a strong electric field," Phys. Rev. B, Condens. Matter, vol. 46, no. 20, pp. 13 100-13 103, Jul. 1992.

[7] B. Cleve, B. Hartenstein, S. D. Baranovskii, M. Scheidler, P. Thomas, and H. Baessler, "High-field hopping transport in band tail of disordered semiconductors," Phys. Rev. B, Condens. Matter, vol. 51, no. 23, pp. 16705-16713, Jan. 1995.

[8] S. D. Baranovskii and P. Thomas, "Non-linear hopping transport in band tails," J. Non-Cryst. Solids, vol. 198-200, pt. 1, pp. 140-145, May 1996.

[9] F. Jansson, S. D. Baranovskii, F. Gebhard, and R. Osterbacka, "Effective temperature for hopping transport in a Gaussian density of states," Phys. Rev. B, Condens. Matter, vol. 77, no. 19, pp. 195 211-195 217, May 2008.

[10] A. Miller and E. Abraham, "Impurity conduction at low concentrations," Phys. Rev., vol. 120, no. 3, pp. 745-755, Jun. 1960.

[11] V. Ambegaokar, B. I. Haperin, and J. S. Langer, "Hopping conductivity in disordered systems," Phys. Rev. B, Condens. Matter, vol. 4, no. 8, pp. 2612-2620, May 1971.

[12] G. E. Pike and C. H. Seager, "Percolation and conductivity: A computer study. I," Phys. Rev. B, Condens. Matter, vol. 10, no. 4, pp. 1421-1434, Sep. 1974.

[13] W. F. Pasveer, J. Cottaar, C. Tanase, R. Coehoorn, P. A. Bobbert, P. W. M. Blom, D. M. de Leeuw, and M. A. J. Michels, "Unified description of charge-carrier mobilities in disordered semiconducting polymers," Phys. Rev. Lett., vol. 94, no. 20, pp. 206 601-206 604, May 2005.

[14] C. Tanase, E. J. Meijer, P. W. M. Blom, and D. M. de Leeuw, "Unification of the hole transport in polymeric field-effect transistors and light-emitting diodes," Phys. Rev. Lett., vol. 91, no. 21, pp. 216 601-216 604, Nov. 2003.

[15] N. I. Craciun, J. Wildeman, and P. W. M. Blom, "Universal arrhenius temperature activated charge transport in diodes from disordered organic semiconductors," Phys. Rev. Lett., vol. 100, no. 5, pp. 56601-56604, Feb. 2008. 